\title{
Fibromyalgia syndrome: still a medical dilemma
}

\author{
P. Sarzi-Puttini ${ }^{1}$, F. Atzeni ${ }^{1}$, S. Perrot ${ }^{2}$ \\ ${ }^{1}$ Rheumatology Unit, L. Sacco University Hospital, Milan, Italy; \\ 2Service de Médecine Interne et Thérapeutique, Consultation de la Douleur, \\ Hôtel Dieu Hospital, Université Paris Descartes, Paris, France
}

$\mathrm{T}$

his issue of "Reumatismo" deals with a variety of aspects of chronic widespread pain (CWP) and its rheumatological quasi-synonym of fibromyalgia (FM) (1). The definitions of CWP and FM both have a long history. Chronic musculoskeletal pain conditions are universally prevalent in both genders and all age groups and, although their pathophysiology varies, they are inter-related anatomically and by their association with pain and impaired physical function. However, there is still no consensus as to whether FM should be considered a discrete entity or just the tip of the iceberg of CWP (2).

Topography can be used if the pain is localised (e.g. biceps tendinitis) and regional syndromes are limited to a body quadrant (myofascial pain syndrome) or cavity (visceral pain referral syndromes), whereas CWP is an accepted term if the pain is diffuse and there are few ancillary symptoms, and FM should be used if the pain is associated with many symptoms or functional syndromes.

The generalised category of CWP implies a systemic process that globally affects the musculoskeletal system. Furthermore, CWP and (particularly) FM are different from localised pain conditions not only in terms of their bodily distribution, but also because of their different impact on people's lives $(2,3)$ as multiple pain sites are associated with more intense and longerlasting pain, more severe disability, and a higher incidence of anxiety and depression. When recording a patient's clinical history, it soon becomes clear that it can take years for full-blown chronic pain to develop, and that the speed of onset of diffuse pain is often related to stressful events or psychoaffective problems.

Although they can be considered synonyms, CWP and FM also have different epidemiological profiles (2). Wolfe et al. [4] observed that the number of patients who meet the criteria for FM is about $2 \%$ of the whole population or two-tenth of those with CWP (10\% of the population), which raises the question of what is the appropriate diagnosis for the remaining $8 \%$. FM may be the intersection of a considerably abnormal and reduced pain threshold with a series of clinical distress variables that include pain, fatigue, sleep disturbance, anxiety and depression. Empirically, central sensitisation can be characterised by using functional brain imaging to demonstrate increased pain processing or by means of experimental pain and sensory testing. The pain associated with central sensitisation has a number of clinical characteristics (5). It tends to be multifocal, widely distributed, and associated with a long (sometimes life-time) history of pain. It is typically familial, more prevalent among females, and frequently initiated by various "triggers" such as early-life stressful events, physical trauma, catastrophic events, infections and peripheral pain syndromes, including inflammatory joint disorders $(5,6)$. FM can also be suspected in the presence of common comorbidities, and clinicians should ask about CWP when faced by patients with conditions such as mood or anxiety disorders that can precede the development of FM. Other common comorbidities in patients with FM include regional pain syndromes that may share certain pathophysiological, including irri-
Corresponding author: Piercarlo Sarzi-Puttini Senior Consultant and Head of Department Rheumatology Unit L. Sacco University Hospital Via G.B. Grassi, 74 - 20127 Milano, Italy E-mail: sarzi@tiscalinet.it 
table bowel syndrome, tension-type headache/migraine, interstitial cystitis or painful bladder syndrome, chronic prostatitis or prostadynia, temporomandibular disorders, chronic pelvic pain, and vulvodynia.

The 1990 American College of Rheumatology Research Classification Criteria (1990 ACR RCC) made FM easier to diagnose (7), but various limitations led to them being replaced by the 2010 criteria (8), which replaced the physical examination item of tender points by the widespread pain index (WPI), a 0-19 count of the number of body regions reported to be painful, introduced a 0-3 severity scale for a series of symptoms characteristic of FM (fatigue, unrefreshing sleep, cognitive problems, and the extent of somatic symptom reporting), which were combined to give a 0-12 symptom severity (SS) scale. (8). The authors combined the SS scale and the WPI to recommend a new case definition of fibromyalgia: (WPI $>$ or $=7$ and $\mathrm{SS}>$ or $=5$ ) or (WPI 3-6 and SS $>$ or $=9$ ).

The ability to evaluate and measure FM as a condition is likely to provide a number of benefits, including the identification of treatment responders in clinical trials and clinical practice. When evaluating the effectiveness of FM therapy, it is important to be able to assess its impact on all of the domains considered important by clinicians and patients, and the OMERACT (Outcome Measures in Rheumatology Fibromyalgia Syndrome Workshop) has recently attempted to include the patient perspective in identifying and prioritising such domains (9).

The use of patient-reported outcomes is becoming an increasingly accepted method of evaluating disease states and management strategies in clinical trials, especially in the case of chronic pain conditions. In agreement with OMERACT, we found that three domains (pain, fatigue and sleep) could be used to create a self-administered Fibromyalgia Activity Score (FAS) for evaluating the efficacy of treatment in FM patients (10).

Another issue is that all three of the pharmaceutical agents approved by the American Food and Drug Administration (FDA) adequately manage only about $50 \%$ of FM patients. Effective therapy may ultimately depend on the biological identification of subgroups of patients who can be expected to respond differently to each of a number of mechanism-related interventions (11). FM treatment requires an individualised approach that often involves multimodal, multidisciplinary management. A number of pharmacotherapies are available that are supported by clinical trial data showing their efficacy in treating pain and other symptom domains, and these should be combined with non-pharmacological modalities such as cognitive behavioural therapy and exercise (12).

\section{REFERENCES}

1. Russell IJ. Fibromyalgia syndrome: presentation, diagnosis, differential diagnosis. Primary Psychiatry, 2006; 13: 40-5.

2. Sarzi-Puttini P, Atzeni F, Mease P. Chronic widespread pain or fibromyalgia? That is the question. Best Pract Res Clin Rheumatol. 2011; 25: 131-2.

3. Ablin JN, Buskila D, Van Houdenhove B, Luyten P, Atzeni F, Sarzi-Puttini P. Is fibromyalgia a discrete entity? Autoimmun Rev. 2012; 11: $585-8$.

4. Wolfe F, Anderson J, Harkness D, Bennett RM, Caro X, Goldenberg DM, et al. Work and disability status of persons with fibromyalgia. J of Rheumat 1997; 24: 1171-8.

5. Sarzi-Puttini P, Atzeni F, Mease PJ. Chronic widespread pain: from peripheral to central evolution. Best Pract Res Clin Rheumatol. 2011; 25: 133-9.

6. Atzeni F, Cazzola M, Benucci M, Di Franco M, Salaffi F, Sarzi-Puttini P. Chronic widespread pain in the spectrum of rheumatological diseases. Best Pract Res Clin Rheumatol. 2011; 25: 165-71.

7. Wolfe F, Smythe HA, Yunus MB, Bennett RM, Bombardier C, Goldenberg DL et al. The American College of Rheumatology 1990 criteria for the classification of fibromyalgia. Arthritis Rheum 1990; 33: 160-172.

8. Wolfe F, Clauw DJ, Fitzcharles MA, Goldenberg DL, Katz RS, Mease P, et al. The American College of Rheumatology preliminary diagnostic criteria for fibromyalgia and measurement of symptom severity. Arthritis Care Res (Hoboken). 2010; 62: 600-10.

9. Mease PJ, Clauw DJ, Christensen R, Crofford LJ, Gendreau RM, Martin SA, Simon LS, Strand V, Williams DA, Arnold LM; OMERACT Fibromyalgia Working Group. Toward 
development of a fibromyalgia responder index and disease activity score: OMERACT module update. J Rheumatol. 2011; 38: 1487-95.

10. Salaffi F, Sarzi-Puttini P, Girolimetti R, Gasparini S, Atzeni F, Grassi W. Development and validation of the self-administered Fibromyalgia Assessment Status: a disease-specific composite measure for evaluating treatment effect. Arthritis Res Ther. 2009; 11 (4): R125.
11. Sarzi-Puttini P, Buskila D, Carrabba M, Doria A, Atzeni F. Treatment Strategy in Fibromyalgia Syndrome: Where Are We Now? Semin Arthritis Rheum. 2008; 37: 353-65.

12. Sarzi-Puttini P, Atzeni F, Salaffi F, Cazzola M, Benucci M, Mease PJ. Multidisciplinary approach to fibromyalgia: what is the teaching? Best Pract Res Clin Rheumatol. 2011; 25 : 311-9. 